\title{
Tras la Puerta de Córdoba
}

\author{
Ricardo Lineros \\ Arqueólogo \\ Director del Museo de la Ciudad de Carmona
}

Una vez terminada la intervención en la Puerta de Córdoba por parte del IAPH, el presente artículo trata de esbozar algunas reflexiones desde la perspectiva local sobre la filosofía, métodos y ámbitos para la puesta en valor de la Puerta de Córdoba.

La finalidad de toda intervención en el Patrimonio Histórico no es otra que la de realizar las funciones sociales que le corresponde. Con frecuencia identificamos, desde el mundo del patrimonio histórico, la idea de puesta en valor con la intervención material, como si los propios valores fueran en sí mismos evidentes e incontrovertibles.

En una sociedad democrática, más allá de toda norma, el Patrimonio Histórico es ante todo un valor social. En este mismo sentido se expresa el preámbulo de la Ley 16/1985 de Patrimonio Histórico Español I: "Su valor lo proporciona la estima que, como elemento de identidad cultural, merece a la sensibilidad de los ciudadanos. Porque los bienes que lo integran se ha convertido en patrimoniales debido exclusivamente a la acción social que cumplen, directamente derivada del aprecio con que los mismos ciudadanos los han ido revalorizando." Como en el resto de los sectores de la sociedad, la normativa tiene que ir precedida y acompañada de su asunción social, de la incorporación de los valores patrimoniales en la vida ciudadana.

Por otra parte, la rápida evolución de las teorías sobre patrimonio histórico a lo largo del siglo $X X$ ha alejado los conceptos y valoraciones utilizados por los especialistas de los del conjunto de la sociedad. Esta distancia no es homogénea y depende, además de otros factores, de la formación y de la emulación de valores o estéticas considerados de prestigio por las elites culturales y/o económicas. Desde esta desigual perspectiva, la puesta en valor del patrimonio histórico no es una tarea sencilla, no porque las teorías sobre el patrimonio histórico sean complejas o difíciles de explicar, ni por la diversidad de interpretaciones y valoraciones que podemos realizar sobre el mismo, sino por la conjunción de todos estos elementos en cada individuo en particular y en suma en el conjunto de la sociedad.

Esta complejidad, derivada de la suma de valoraciones, interpretaciones, experiencias, emociones, etc., da lugar ocasionalmente a paradojas, de tal modo que actuaciones dirigidas a la valoración del patrimonio pueden dar lugar a resultados perversos y contrarios.

En consecuencia, en nuestra opinión, al abordar la puesta en valor de la Puerta de Córdoba es imprescindible tomar en consideración la percepción de la misma por los vecinos más próximos, por el conjunto de los carmonenses, por los visitantes etc.

Acerca de este tema, señalaremos un ejemplo reciente: La intervención por parte de la Oficina Municipal de Rehabilitación, dentro del programa de transformación de infravivienda de la Consejería de Obras Públicas, en una casa de vecinos de la calle Caño Quebrado número 2, situada al interior del Conjunto Histórico de Carmona. El programa, en síntesis, consiste en la rehabilitación de casas de vecinos cuyos inquilinos son alojados en otras casas durante las obras, para, una vez finalizadas, volver a ocuparlas. El inmueble en cuestión reveló, bajo una epidermis de cal y sobrepuestos, una arquitectura mudéjar de la que destacaban formalmente varios arcos apuntados enmarcados en alfiz y fábrica de ladrillo, que fueron correctamente integrados, así como el mantenimiento de otros elementos, tales como forjado de madera, etc.. Pues bien, durante el realojo de los vecinos, algunos mostraron su desencanto con el mantenimiento de las vigas de madera, los muros sin enlucir, etc. La explicación la encontramos en el párrafo anterior- 
La pluralidad de miradas desde las que podemos acercarnos a la puesta en valor de la Puerta de Córdoba es extraordinariamente amplia. Por el contrario, la difusión de sus significados conviene realizarla desde una visión integradora de lo patrimonial y desde un proyecto de idea de ciudad.

mente citado del preámbulo de la LPHE resumido en dos palabras: identidad y aprecio. Identificaban estos elementos con una situación de carencia y de pobreza y en consecuencia la respuesta emocional no podía ser otra que la falta de aprecio hacia los elementos patrimoniales. La estrategia que seguimos fue incluirlo en el programa de visitas de las Jornadas Europeas del Patrimonio 2000, dedicadas al mudéjar, en detrimento de otros inmuebles de reconocida monumentalidad y estética, para que, además de las explicaciones, las visitas reforzasen las valoraciones patrimoniales de los inquilinos y del barrio.

En el caso de la Puerta de Córdoba, frente a la arquitectura popular mudéjar, disponemos de una ventaja aparente: la de su percepción y valoración por todos como monumento. Además de una categoría legal, la palabra monumento tiene una significación común basada en cualidades estéticas o de grandes proporciones. Ahora bien, ies esta idea de monumento la que más conviene a la hora de su puesta en valor? Creemos que no, y así lo avala la actual teoría de los bienes culturales al señalar el valor del entorno, al que aquí hacemos referencia, no ya como figura de protección, sino en su estricto sentido de contexto. Contexto físico y material constituido por inmuebles y objetos muebles, e inmaterial como la memoria, la tradición oral o literaria etc., y que en definitiva caracterizan su interpretación histórica.

Obsérvese en este punto, que en las últimas líneas hemos pasado de la idea común y generalizada de patrimonio histórico a otra de carácter legal y profesional -entorno, contexto ... -.

La idea de patrimonio es aún más compleja en cuanto que en las últimas décadas del siglo $X X$ han emergido con fuerza nuevas funciones sociales junto a las tradicionales educativas y culturales, como las de cohesión social, de desarrollo, económicas, etc.-La dificultad es mayor si tenemos en cuenta que las percepciones del concepto y valores del patrimonio no tienen una aceptación armónica y lineal en todas su acepciones en el conjunto de la sociedad. Así, por ejemplo, ha sido más rápida la aceptación del patrimonio histórico como producto de demanda del turismo cultural, frente a su reconocimiento como recurso básico para la innovación y creación en el sector de las industrias culturales.

Desde el punto de vista de la puesta en valor del patrimonio histórico, en nuestro caso la Puerta de Córdoba, no podemos estar ajenos a las transformaciones del propio concepto y sus funciones sociales, y al papel cada vez más determinante de la difusión y comunicación.

Ahora bien, ¿en qué espacio tiene lugar esa confrontación de conceptos e ideas y su materialización en iniciativas y acciones?. Sin duda, en el ámbito local.

Sin embargo, llama la atención la completa ausencia en las Administraciones Locales de oficinas y unidades administrativas especializadas y dedicadas al patrimonio histórico, en comparación con la asunción de otros servicios como los sociales, promoción y desarrollo, de empleo y formación, etc. Puede objetarse a estas consideraciones, de un lado la actividad de las Oficinas de Urbanismo, consideradas habitualmente como las más próximas, dada su incidencia a través del planeamiento en la conservación y protección del patrimonio histórico, pero ésta representa sólo un segmento de toda la problemática actual del patrimonio histórico; y de otro la de las Oficinas de Cultura cuyas actividades han estado dirigidas más bien a la promoción del teatro, del cine, de talleres de expresión, etc. En definitiva, las Administraciones Locales se han considerado ajenas a la gestión del patrimonio histórico a pesar de ser en la actualidad un tema transversal a la formación, el empleo, la cohesión y promoción social, el desarrollo económico etc. La Ley 1/199| de Patrimonio Histórico Andaluz, no obstante, encomienda a los Ayuntamientos en su artículo $4^{\circ}$ "realizar y dar a conocer el valor cultural de los bienes integrantes del Patrimonio histórico Andaluz, que radiquen en su término municipal." -recuerden que el patrimonio histórico es ante todo un valor social-. 
Sin duda, tras la llegada de la democracia, la carencia de una legislación adecuada a los tiempos motivó una atención preferente al desarrollo de los mecanismos de conservación y protección sobre la difusión. Subrayaremos en este punto, que a nuestro juicio, una vez instituida la tutela y desarrollado sus instrumentos, la realización de las funciones sociales del patrimonio histórico de un modo equilibrado, requiere una disposición de mayores facultades para la Administración Local, como la más próxima, y el fomento de la participación ciudadana.

Tras estas consideraciones, analizaremos el significado de la Puerta de Córdoba desprendiéndonos de todo atributo específico. El término puerta implica entrar y salir, la existencia de un espacio acotado, la elección de un lugar, la preexistencia de elementos formadores, etc.; desde el análisis arqueológico la superposición de unidades de estratificación, que a su vez implican lapsus de tiempos, etapas, etc.; desde un punto de vista etnográfico, la existencia de ritos, la consagración del lugar, etc; desde el arte ... ; desde la arquitectura...; etc. La pluralidad de miradas desde las que podemos acercarnos a la puesta en valor de la Puerta de Córdoba es extraordinariamente amplia. Por el contrario, la difusión de sus significados conviene realizarla desde una visión integradora de lo patrimonial y desde un proyecto de idea de ciudad. Desde esta perspectiva, la apertura de una sala en la que se exhiba su historia o la integración parcial de tal o cual elemento no son actuaciones suficientes. Como metodología, creemos más adecuada la de considerar a la ciudad histórica como museo, y a la puerta como un elemento más a musealizar. La aplicación de estos conceptos para la transmisión de significados e interpretaciones tiene la virtualidad de contemplar la conservación, la investigación y la difusión como un único proceso.

Sin ánimo de incorporar a estas reflexiones nuevos campos de debate que, en sí mismos, no son sustantivos para el tema que tratamos, en nuestra opinión, en la era de las comunicaciones y la globalización, una evolución verosímil del concepto y actividades de los museos, además de las tareas tradicionales de conservar y exhibir los elementos materiales, será la de convertirse en verdaderos centros de comunicación y servidores de la cultura (-solo se conserva aquello que se comunica- ).

Ahora bien, ¿conservación de qué?, del inmueble, del entorno, de los objetos muebles asociados, de la cultura inmaterial, etc. La idea de conservación es tan compleja como el concepto de patrimonio histórico y las funciones que desempeña. La vinculación de la conservación al concepto de comunicación le obliga a desenvolverse en un contexto dinámico y versátil, en el que el tiempo de respuesta da credibilidad a sus significados.

La idea de conservación de la Puerta de Córdoba no debe descansar en la realización de un proyecto ejemplar de restauración cada diez o cincuenta años. La mejor garantía para la conservación de la
Puerta de Córdoba es la de la convivencia con sus vecinos, de modo que estos y los carmonenses en general sigan viendo en ella un elemento de identificación. El método inmediato es la pedagogía y la demostración de todas las funciones sociales que hoy somos capaces de reconocer en el patrimonio histórico, y de este modo, acercar la conservación a lo cotidiano, a la idea de mantenimiento, y a una idea de mantenimiento que no parte de la norma, sino de la patrimonialización de la misma por sus convecinos, es decir la consideración de lo público como algo que pertenece sensiblemente a cada individuo. En definitiva, se trata de fomentar una forma de cultura y de entender la cultura.

Al igual que la conservación, la investigación y difusión están sujetas a la evolución de las teorías sobre los bienes culturales y al reconocimiento de las funciones sociales que estos desempeñan.

La difusión, en los últimos años ha experimentado un creciente interés a medida que se produce el traslado del centro de atención del objeto al sujeto. La evolución de nuestro entorno social y económico nos permite suponer una rápida evolución de la idea de difusión y un incremento de las acciones y medios utilizados para tal fin.

Ahora bien, la evolución de los conceptos, la aparición de nuevas funciones o modos de difusión, o la aplicación de innovadoras metodologías, suponen nuevos riesgos para el patrimonio histórico, entre los que son más evidentes la imposición de modelos uniformes o su conversión en un producto de consumo carente de los valores educativos y culturales, que les son propios. El diagnóstico y cauterización de estos riesgos requiere una atención preferente a la investigación y a la formación, sobre todo, con la implicación de las Administraciones Locales, ya que las causas y elementos que dinamizan las transformaciones están en su campo de acción.

En este sentido, conviene la promoción de la investigación en una doble vertiente:

- investigación científica, técnica y experimental, desde la pluralidad de disciplinas y metodologías.

- investigación aplicada al diseño e innovación de productos culturales, que satisfagan la demanda social, con contrastada calidad y autenticidad.

En cuanto a la formación, además de la superior o de postgrado, -especializada e interdisciplinar-, las principales carencias están en la esfera de la fomación profesional y ocupacional.

Finalmente, señalar que la garantía de un desarrollo adecuado del patrimonio histórico, desde el punto de vista de la conservación, de la investigación y la difusión, deviene de abordar estas como partes de un mismo proceso. En este sentido la cooperación entre los profesionales del patrimonio y las distintas administraciones es esencial. 
TIPO DE ACTUACIÓN:

Restauración de la Puerta de Córdoba en Carmona (Sevilla)

PROPIETATARIO DEL B.I.C::

Ayuntamiento de Carmona

PROMOTOR:

Delegación Provincial en Sevilla de la Consejería de Cultura.

REALIZACIÓN:

Instituto Andaluz del Patrimonio Histórico. Dirección General de Bienes Culturales.

Consejería de Cultura de la Junta de Andalucía.
DIRECCIÓN:

R. Fernández-Baca Casares, Director del I.A.P.H.

COORDINACIÓN GENERAL DEL PROYECTO:

L. Pérez del Campo, Jefe del Centro de Intervención. I.A.P.H.

PROYECTO Y DIRECCIÓN TÉCNICA:

A. Tejedor Cabrera, arquitecto.

RESPONSABLE DE ANÁLISIS Y ENSAYOS:

R. Villegas Sánchez. Jefe del Dpto. Análisis del I.A.P.H.

RESPONSABLE DE PROYECTO Y ESTUDIOS HISTÓRICOS:

R. Ojeda Calvo. Dpto. de Investigación del I.A.P.H.

\section{Equipo técnico}

\section{ANÁLISIS DE MATERIALES Y ENSAYOS:}

Rosario Villegas Sánchez, Jesús Espinosa Gaitán, Francisco Gutiérrez Montero, Lourdes Martín García y Esther Ontiveros Ortega.

\section{COLABORADORES:}

José Luis de Justo Alpañés (A.I.C.I.A. e Instituto Universitario de Ciencias de la construcción-E.T.S.A. de Sevilla).

\section{INVESTIGACIÓN HISTÓRICO-ARQUEOLÓGICA:}

Reyes Ojeda Calvo, Antonio Pérez Paz y Miguel Angel Tabales Rodríguez.

\section{COLABORADORES:}

Arqueólogos:

Arturo Pérez Plaza, Eduardo Prados Pérez y Ana Romo Salas,

Estudios paleobiológicos:

Eloisa Bernáldez Sánchez y María Bernáldez Sánchez

Trabajos de delineación:

Juan Antonio Estepa Rosado y Miguel Angel Hernández

Dibujos de la puerta romana:

Francisco Salado Fernández

Ensayos de restitución en 3D:

Antonio Pérez Paz y José $M^{a}$ Rodrigo Cámara

INVESTIGACIÓN DOCUMENTAL Y ESTUDIO HISTÓRICOARTÍSTICO:

Juan Antonio Arenillas Torrejón

\section{COLABORADORES:}

Historiador del Arte

Gabriel Ferreras Contreras

Arquitecto

Alfonso Jiménez Martín

\section{Bibliografía generada}

Bernáldez Sánchez, E.: "El nicho ecológico de la Paleobiología en el Patrimonio Histórico", P.H. Boletín del Instituto Andaluz del Patrimonio Histórico. Año IV, n 16 (septiembre 1996) pp. 48-59.

Espinosa, J.; Gutiérrez, Fi; Villegas, R.

"Previous studies for the restoration of the Cordoba Door, Carmona (Spain)". 8th Int. Cong on Deterioration and conservation of stone. Pp. 100 I-1008, Berlín, 1996

Espinosa, J., Ontiveros, E., Villegas, R., Alcalde, M.: "Evaluation of treatments for the stone of the Cordoba Door, Carmona (Seville, Spain)". Proc. 5th Int. Symp. The conservation of monuments in the Mediterranean Basin. Sevilla 2000. En prensa.

Ojeda Calvo, R.: "Nuevos datos sobre la Puerta de Córdoba en época romana". En II Congreso de Historia de Carmona "Carmona Romana" (Carmona- 29 de septiembre a 2 de octubre de 1999).

\section{COLABORADORES:}

\section{Arquitectos}

Mercedes Linares Gómez del Pulgar, Pedro Lobato Vida

Arquitecto técnico

Ángel González Martín

Delineación:

$M^{\mathrm{a}}$ José Llanos, Carlos de Miguel y Daniel Plaza Gómez.

Levantamiento taquimétrico:

Carlos Encina Lobo

Estudio estratigráfico de enlucidos:

Fátima Bermúdez-Coronel $\mathrm{G}^{\mathrm{a}}$ de Vinuesa y $\mathrm{M}^{\mathrm{a}} \mathrm{Paz}$ Ruiz del

Portal Ruiz-Granados

Maqueta:

Jacinto Gómez Piris y María González García

\section{EMPRESA CONSTRUCTORA:}

U.T.E. Necso-Freyssinet

RESTAURACIÓN LIENZO DE LA VIRGEN DE GRACIA:

Lucía Sameño Puerto, restauradora.

\section{FOTOGRAFÍA:}

Eugenio Fernández, José Manuel Santos Madrid, Fernando Alda Calvo y los autores de la actuación.

\section{OTRAS COLABORACIONES:}

Exmo. Ayuntamiento de Carmona, Rainiero Baglioni, Ana Bouzas Abad, Amalia Cansino Cansino y $M^{\mathrm{a}}$ José González Fernández, restauradores.

\section{AGRADECIMIENTOS:}

D. Brooke Alexander, D. Fernando Amores Carredano, D. Antonio Caballos Rufino, D. Francisco López González, Dña. Ana Lorca Valenzuela, Dña. Ma Ángeles Piñero Márquez, Dña. Ma Dolores Rivas Roldán, D. José Manuel Rodríguez Hidalgo.

Nuestro agradecimiento especial al Excmo. Ayuntamiento de Carmona.
Ecxmo. Ayto. de Carmona-Univ. de Sevilla-Consejería de Cultura. En prensa.

Ojeda Calvo, R. y Tabales Rodríguez, M.A.: "La investigación arqueológica en Bienes Inmuebles. Metodología aplicada en la Puerta de Córdoba de Carmona", P.H. Boletín del Instituto Andaluz del Patrimonio Histórico. Año IV, nº I5 (junio 1996) pp. 4I-52.

Ojeda Calvo, R. y Pérez Paz, A.: “Metodología aplicada a la intervención arqueológica en bienes inmuebles: hacia un modelo de registro y gestión de datos", P.H. Boletín del Instituto Andaluz del Patrimonio Histórico. Año IV, n 17 (diciembre 1996) pp. 50-58.

Tejedor Cabrera, A.:"'El proyecto de restauración de la Puerta de córdoba en Carmona", P.H. Boletín del Instituto Andaluz del Patrimonio Histórico. Año Vl, nº 22 (marzo 1998) pp. 50-66. 